\title{
Modeling of Complex and Freeform Surfaces by using Reverse Engineering Approach
}

\author{
Y. P. Jadhav ${ }^{1}$, V. N. Chougule ${ }^{1}$, A. V. Mulay ${ }^{2}$ \\ ${ }^{1}$ (Department of Mechanical Engineering, M.E.S. College of Engineering, Pune, Maharashtra, India) \\ ${ }^{2}$ (Department of Production Engineering \& Industrial Management, College of Engineering, Pune, \\ Maharashtra, India)
}

\begin{abstract}
Reverse Engineering is extensively used technology by both research and industries for rapid development of product. In Reverse engineering with the help of already, existing product a new product is developed. To develop a new product CAD model is necessary. For this purpose already existing object is scanned using CMM machine, laser scanner etc. Output of Scanner is Point cloud data. It is big challenge to get CAD model from point cloud data. Various techniques called segmentation technique are available to convert point cloud in to surface or solid geometry. This paper reviews the reverse engineering and various segmentation techniques from the literature. Case study carried out to convert point cloud data in to CAD model by using FreeCAD Software.
\end{abstract}

Keywords -CAD, Reverse Engineering, Point cloud data, Segmentation

\section{INTRODUCTION}

In recent year, there is rapid change in the manufacturing process; most of traditional manufacturing processes replaced by Computer Aided Manufacturing (CAM). For CAM, there is necessity of Computer Aided Design (CAD) model. Different software's are available in the market to develop a cad model of the product. However, to use this method we should know all the dimensions of product. CAD model can obtain by using reverse engineering approach. It is helpful in industries to develop a new product using the already existing product. In reverse engineering, existing product scanned to get point cloud data. CAD model can generated from this data by using segmentation technique. CAD model develop by RE approach further used for Rapid prototyping (RP). So accuracy of manufactured product is totally depends on the accuracy of the CAD model developed. For better output highly accurate model should develop. Accuracy of CAD model is depends on the accuracy of scanning and Segmentation technique. For this purpose it is necessary to overview all the segmentation techniques which gives the better results. In addition, there are number of software's available in the market, which can convert point cloud in to CAD model.

\section{LITERATURE SURVEY}

C.K. Au and M.M.F. Yuen [1] propose a feature-based reverse engineering method for mannequin in garment design. An automated reverse engineering approach for human torsos creates accurate parameterized models. A key concept in their method is creating a generic mannequin model of a human torso appropriately aligned with the $3 \mathrm{D}$ point cloud of the desired human torso model. Woo, et al. [2], a new method for segmenting the point cloud data is proposed. The proposed algorithm uses the octree-based 3D-grid method to handle a large amount of unordered sets of point data. The final 3D-grids constructed through a refinement process and iterative subdivision of cells using the normal values of points. This 3D-grid method enables us to extract edgeneighborhood points while considering the geometric shape of a part. Ke, et al. [3] presented two integrated solution schemes, sectional feature based strategy and surface feature based strategy, for modeling industrial components from point cloud to surfaces without using triangulation. For the sectional feature based strategy, slicing, curve feature recognition and constrained fitting introduced. The surface feature based strategy relies on differential geometric attributes estimation and diverse feature extraction techniques. The methods and algorithms such as attributes estimation based on 4D Shepard surface, symmetry plane extractions, quadric surface recognition and optimization, extruded and rotational surface extraction, and blend feature extraction with probability and statistic theory are proposed. J. Roca-Pardiñas, et al. [4] presented a new method for the reconstruction of three-dimensional objects surveyed with a terrestrial laser scanner. The method is a $2.5 \mathrm{D}$ surface modeling technique which is based on the application of statistical nonparametric regression methods

$5^{\text {th }}$ National Conference RDME 2016, 10-11 $1^{\text {th }}$ March 2016. 
for point cloud regularization and mesh smoothing, specifically the kernel-smoothing techniques. The proposed algorithm tested in a theoretical model-simulation carried out with the aim of evaluating the ability of the method to filter random noise and oscillations related to the acquisition of data during the fieldwork. Herbert J. Koelman [5] presented an industrial application of CAD in which concern the measurement and reengineering of the shape of a complete ship hull and of ship's parts, which is a frequently recurring task in the shipbuilding and ship repair sector. One of the considerations in this respect was that with photogrammetry not only the 3D geometry could measure, but that also topological properties will implicitly take. Therefore, a reengineering system developed, which consists of two major parts: the shape processing software and the photogrammetric measurement, which tightly coupled. This system has proved to work fine for large-scale 3D objects. Enrico Vezzetti [6] performed morphological analysis of a surface cannot carry out without subdividing the point cloud into subsets characterized by the same morphological complexity. There are many different solutions, strongly correlated with the specific context where they can apply. Firstly, a variable set, composed by acquired geometry and the acquisition device parameters, has introduced in order to describe the possible working conditions that users. Jin Liua, Zhong-ke Wuaa [7] presented adaptive approach for primitive shape detection from point clouds presented. Approach based on RANSAC primitive shape detection algorithm, and makes the primitive shape detection procedure adaptive by histogram analysis of point's deviation from their corresponding fitted primitive shapes. YutaoWanga,et al. [8] proposed a robust normal estimation method to estimate normal for noisy point clouds with outliers. The proposed method incorporates three weights effectively reduce the influence of outliers both in the spatial and in the normal field on the normal estimation. In addition, the proposed method is applicable to points in smooth regions as well as around sharp features via employing locally adaptive bandwidth parameters for the weights. Atul Kumar, [9] proposed that K-means algorithm has good ability to handle the large number of scanned data. It is best suited for creation a desired shape curve-likes shape for near-best approximation of the scanned data point set. Reconstructing the smooth curve achieved by proposed algorithm in MATLAB environment. Gabor Erdos [10], presented CAD model matching method takes widely supported input in form of mesh models, works also for partially measured objects, and for any type of features. It only needs an appropriate distance function for assessing the degree of match between a feature and its respective point cloud data. Tolerance of matching can adjust, so the method is capable of handling noisy data.

\section{PhASES OF REVERSE ENGINEERING}

\section{A. Data Capture}

This phase includes the deciding the proper scanning technique and actual scanning of object to get information about all the features of the object. All the scanning devices give the output in form of point cloud data. There are two types of scanning devices, Contact and non-contact type. In contact type devices, there is direct contact between object and Scanner probe E.g. Coordinate measuring machine. In non-contact type devices, there is no direct physical contact E.g. Optical scanner, Laser scanner etc. Mostly non-contact type devices are preferred.

\section{B. Preprocessing of Data}

This phase includes reducing noise from collected data, point reduction and merging of multiple data sets. Predefined filters used for noise and point reduction. To improve the quality of data multiple data sets taken and merged together. This phase gives the merged, clean point cloud data.

\section{Triangulation}

In triangulation process, polygon mesh can easily create for given point cloud data. Delaunay triangulation is the concept behind this process. There are many algorithm exists for triangulation including alpha shapes, marching cubes, ball pivoting algorithm, , moving least squares Poisson surface reconstruction etc.

\section{Segmentation}

Segmentation is the most important and complex step of the reverse engineering. Segmentation groups the original data points or mesh into subsets each of which logically belongs to a single primitive surface.

Segmentation process, involves a fast algorithm for k-nearest neighbors search and an estimate of first- and second-order surface properties. The first-order segmentation, which based on normal vectors, provides an initial subdivision of the surface and detects sharp edges as well as flat or highly curved areas. The second-order segmentation subdivides the surface according to principal curvatures and provides a sufficient foundation for the classification of simple algebraic surfaces. 


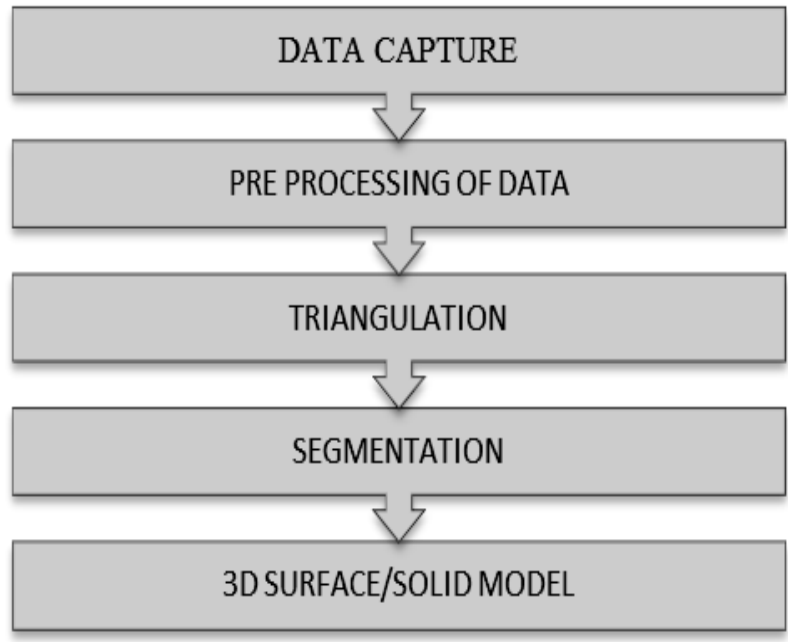

Fig 1: Phases of Reverse Engineering

\section{E. 3D Surface/ Solid Model}

Boundary representation and feature-based are the two basic representations for solid models.

Various methods proposed to construct B-rep models from point clouds or triangular mesh. Some focused on manufacturing feature recognition for process planning purpose. One promising development in recent years was the geometric feature recognition, which automatically recognizes solid features embedded in B-rep models.

1. Boundary Representation: Based on segmented regions, a region adjacent graph built, which reflects the complete topology and serves as the basis for building the final B-rep model, where the individual bounded surfaces glued together along their common edges. Three steps involved in constructing B-rep models, flattening, edges and vertices calculations, and stitching. In flattening step, regions are extended outwards until all triangles have been classified which is necessary to remove gap between regions. Sharp edges can be calculated using surface-surface intersection routines, and vertices where three surfaces meet determined. During the process, a complete B-rep topology tree is also constructed. B-rep model can be created by stitching together the faces, edges, and vertices.

2. Geometric Feature Recognition: B-rep models are not feature-based. In order to convert a B-rep model into a featurebased solid model, the embedded solid features must recognized, and a feature tree that describes the sequence of feature creation must create.

CAD model generated from this method converted in to neutral formats like IGES, STEP. Therefore, we can use these models in any CAD package for further use.

\section{CASE STUdY}

For the case study, point cloud data of Rotor selected and further converted it into CAD model by using FreeCAD software. Free $\mathrm{CAD}$ is a free and open-source general-purpose parametric 3D CAD modeller. Various steps used for this conversion are

- Import the stl file in the "mesh design"

- Clean up your stl using the mesh design tools.

- $\quad$ Switch to the "part" work space

- Click on the stl file in the left plane

- Click on the part menu, scroll down to "create part from mesh" then wait

- $\quad$ Export the solid model as an IGES file.

- Solidworks, Design Magic both will read in the file and allow its manipulation.

•

\section{RESULTS AND DISCUSSION}
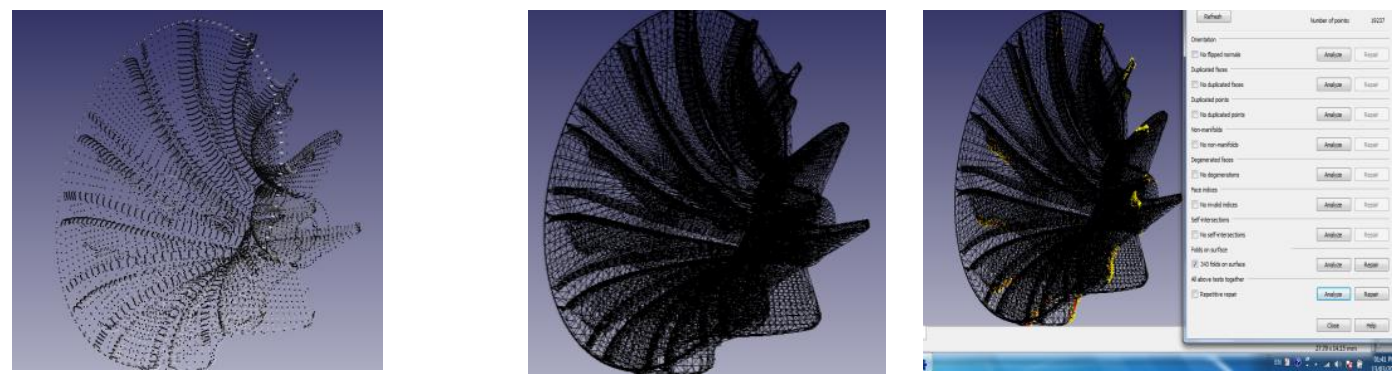
Fig3: Point cloud of rotor

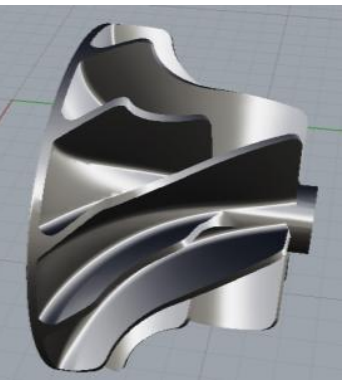

Fig 7: Export to solid Model
Fig 4: Point cloud data in STL format
Fig 5: Edge construction of STL using mesh design tool

This step by step conversion is shown in these Figs. 3-7, here we get CAD model in IGES file format which can further edited for the modifications.

\section{CONCLUSIONS}

In this paper, reverse engineering and segmentation technique explained in detail. Reverse engineering plays vital role in the rapid product development. We can use either available software's or segmentation algorithm to get $\mathrm{CAD}$ from point cloud data. Case study shows easy steps to get CAD model from point cloud data using freeCAD software. Accuracy of CAD depends on quality of cloud data. If there are errors in data, they can directly reflect in CAD model. Therefore, it is difficult to use CAD modeling software's directly. Various algorithms are there to eliminate the data error but still they cannot give higher accuracy. It is necessary to develop algorithms with can eliminate the all type of errors error and give highly accurate models. In addition, these algorithms should be useful for the complex geometries.

\section{REFERENCES}

[1] C.K. Au and M.M.F.Yuen (1999) "Feature-Based Reverse Engineering of Mannequin for Garment Design" Computer Aided design 31,751-759.

[2] H. Woo, E. Kang, Semyung, Wang, Kwan H. Lee (2001) "A new segmentation method for point cloud data" International Journal of Machine Tools \& Manufacture 42:167-178.

[3] Landecir A. Albuquerque, José Mauricio S. T. Motta (2004) "3D Reconstruction of Free-Form Objects from Range Images Acquired by Laser Scanning”. Instituto Nacional de Mathematical Pura E Aplicada, Brazil

[4] Yinling Ke, Shuqian Fan, Weidong Zhu, An Li, Fengshan Liu, Xiquan Shi (2005) "Feature-based reverse modeling strategies" Computer-Aided Design 38:485-506.

[5] J. Roca-Pardiñas, H. Lorenzo, P. Arias b, J. Armestob (2008) "From laser point clouds to surfaces: Statistical nonparametric methods for three-dimensional reconstruction" Computer-Aided Design 40:646-652.

[6] Manzoor Hussain M.1, Sambasiva Rao CH.2 and Prasad K. E (2008) "Reverse engineering: Point cloud generation with CMM for part modeling and error analysis". Asian Research Publishing Network (ARPN) vol 3

[7] Herbert J. Koelman (2010) "Application of a photogrammetry-based system to measure and re-engineer ship hulls and ship parts: An industrial practices-based report” Computer-Aided Design 42:731-743.

[8] Chung-Shing Wang, Wie-Hua A. Wang, Man-Ching Lin(2010) "STL Rapid Prototyping Bio-CAD model for CT medical segmentation" Elsevier journal, 3(61),187-197.

[9] Enrico Vezzetti (2011) "Study and development of morphological analysis guidelines for point cloud management: The decisional cube" Computer-Aided Design 43:1074-1088.

[10] Shaw L, Weckenmann A (2011) "Automatic Registration Method for Hybrid Optical Coordinate Measuring Technology". CIRP Annals - Manufacturing Tech-nology 60:539-542.

[11] Jin Liua, Zhong-ke Wuaa (2012) an adaptive approach for primitive shape extraction from point clouds. Optik 125:2000-2008

[12] L. M. Mestetskiy, N. F. Dyshkant, D. Gordev, M. S. Kumari, B.H. Shekar(2012) "Surface measures for accuracy evaluation in 3D face reconstruction"10(45), 3583-3589.

$5^{\text {th }}$ National Conference RDME 2016, 10-11 $1^{\text {th }}$ March 2016.

M.E.S. COLLEGE OF ENGINEERING, PUNE. 411001

14 Page 
[13] V. N. Chougule, R. N.Nirgude et al(2012) "Fitting Spline Curves through Set of Unorganized Point Cloud Data" IOSR-JMCE PP: $51-55$

[14] Yutao Wanga, Hsi-YungFenga, Félix-Étienne Delormeb, Serafettin Engin (2013) “An adaptive normal estimation method for scanned point clouds with sharp features" Computer-Aided Design 45:1333-1348.

[15] Atul Kumar, P. K. Jain, P. M. Pathak (2014) “Curve Reconstruction of Digitized Surface using K-means Algorithm” Procedia Engineering 69,544-549

[16] Gabor Erdos, Takahiro Nakano, Jozsef Vancza (2014) "Adapting CAD models of complex engineering objects to measured point cloud data" CIRP Annals - Manufacturing Technology 63:157-160. 